\title{
Energy dissipation in head-on collisions of spheres
}

\author{
S Krijt ${ }^{1} \ddagger$, C Güttler ${ }^{2,3}$, D Heißelmann ${ }^{3}$, C Dominik ${ }^{4,5}$ \\ and A G G M Tielens ${ }^{1}$ \\ ${ }^{1}$ Leiden Observatory, Leiden University, P.O. Box 9513, 2300 RA Leiden, The \\ Netherlands \\ ${ }^{2}$ Department of Earth and Planetary Sciences, Kobe University, 1-1 Rokkodai-cho, \\ Nada-ku, Kobe 657-8501, Japan \\ ${ }^{3}$ Institut für Geophysik und extraterrestrische Physik, Technische Universität \\ Braunschweig, Mendelssohnstraße 3, 38106 Braunschweig, Germany \\ ${ }^{4}$ Astronomical Institute "Anton Pannekoek", University of Amsterdam, PO Box \\ 94249, 1090 GE Amsterdam, The Netherlands \\ ${ }^{5}$ Afdeling Sterrenkunde, Radboud Universiteit Nijmegen, Postbus 9010, 6500 GL, \\ Nijmegen, The Netherlands \\ E-mail: krijt@strw.leidenuniv.nl
}

\begin{abstract}
Collisions between spheres are a common ingredient in a variety of scientific problems, and the coefficient of restitution is a key parameter to describe the outcome of those. We present a new collision model that treats adhesion and viscoelasticity self-consistently, while energy losses arising from plastic deformation are assumed additive. Results show that viscoelasticity can significantly increase the energy that is dissipated in a collision, enhancing the sticking velocity. Furthermore, collisions well above the sticking velocity remain dissipative. We systemically compare the model to a large and unbiased set of published laboratory experiments to show its general applicability. The model is well capable of reproducing the important relation between impact velocity and coefficient of restitution as measured in the experiments, covering a wide range of materials, particle sizes, and collision velocities. Furthermore, the fitting parameters from those curves provide physical parameters like the surface energy, yield strength, and characteristic viscous relaxation time. Our results show that all three aspects - adhesion, viscoelastic dissipation and plastic deformation - are required for a proper description of the kinetic energy losses in sphere collisions.

PACS numbers: 45.50.Tn, 46.06.+b, 68.35.-p, 46.35.+z
\end{abstract}

Submitted to: J. Phys. D: Appl. Phys.

$\ddagger$ Corresponding author. Phone $+31(0) 715278452$. 


\section{Introduction}

The study of the normal impact of a sphere and a second sphere or a substrate is a fundamental one, with applications in many different fields and scientific problems. The outcome of a collision is typically described by the coefficient of restitution, the ratio of the relative post- and pre-collision velocities, a measure of the amount of kinetic energy that is lost during the collision. Its value determines the energy balance in collisional systems such as granular gasses [1,2], which find applications in a wide range of physical environments. The question whether or not a collision results in sticking is of importance for many practical problems, such as the transportation of powders or granular materials, as well as for the study of the evolution of agglomerates, for instance in astrophysical environments such as dense molecular clouds [3], protoplanetary disks [4, 5, 6], and planetary rings $[7,8]$. Conversely, a proper description of these systems requires good understanding of the physical processes involved and the material parameters describing them.

During a collision, various physical mechanisms can work together to dissipate kinetic energy. The magnitude of the energy associated with these mechanisms will in general depend on the target and projectile materials, their sizes, and the collision velocity. At low collision velocities, the surface energy of the materials associated with the mutual contact area is comparable to the kinetic energy, and will influence the collisional outcome, often resulting in sticking. In more energetic collisions, adhesion becomes less important, and sticking cannot be achieved through surface forces alone. Energy-loss mechanisms that are dominant in this regime can include the excitation of elastic waves $[9,10]$ and viscoelasticity $[1,11]$. During high impact velocities, the stresses in and around the contact area may become so large that the material stops to behave elastically. Kinetic energy will then go into plastic deformation, and this becomes the most important energy sink [12].

Despite extensive theoretical work studying these underlying physical processes, and experimental studies with measurements of the coefficient of restitution for certain velocities, e.g. $[13,14,15,16,17,18]$, few attempts have been made to combine different experiments and systematically compare the observed coefficient of restitution to theoretical predictions. In general, the experimental results show three different features [19]: (1) A rapidly increasing coefficient of restitution for low velocities, possibly preceded by sticking collisions. (2) A region where the coefficient of restitution does not vary with collision velocity, and has a value that is significantly smaller than unity. (3) At high velocities, the coefficient of restitution is seen to fall of with velocity. A complete model for the collisional outcome has to be able to reproduces all three of these characteristics.

In this work, we set out to create collision model for viscoelastic adhesive spheres, which will be tested against published experimental results. While theoretical studies including either viscoelasticity $[1,11]$, or adhesion $[12,20]$ have been around for some time, combining these two effects has proven notoriously difficult. In section 
2, we propose a dynamic contact model for adhesive viscoelastic spheres. The main advancement of our model is that the two variables that describe the contact the contact radius and the distance between the sphere's centers - are treated as mutually independent, where most theories assume an equilibrium relation between these quantities. The contact description is used in section 3 to describe head-on collisions between spheres, after which it is expanded to include plastic deformation at high velocities. Next, the collision model is compared to a large and unbiased set of collision experiments [19]. Fitting the model to the various experimental results will reveal how well it is able to reproduce the collisional outcomes, and allows to get a handle on the material properties governing the adhesive, viscous, and plastic behavior in question (section 4). The results are discussed in section 5, after which the main conclusions are presented in section 6 .

\section{Contact model}

In the section we briefly revisit elastic contact theory (with and without adhesion), before deriving the equations describing a viscoelastic contact.

\subsection{Static contact}

When two smooth elastic spheres of radii $R_{1}$ and $R_{2}$ are pressed together, they will deform locally and share a circular contact area with radius a (figure 1). Assuming the contact is small compared to the reduced radius $R$, and there are no forces acting

outside the contact area, we can write the pressure distribution in the contact area as a function of $0 \leq r \leq a[21]$

$$
p(r)=\frac{E^{*}}{\pi R} \frac{a^{2}-2 r^{2}+R \delta}{\sqrt{a^{2}-r^{2}}},
$$

where $R^{-1} \equiv R_{1}^{-1}+R_{2}^{-1}$, and $E^{*-1} \equiv\left(1-\nu_{1}^{2}\right) / E_{1}+\left(1-\nu_{2}^{2}\right) / E_{2}$ is the combined elastic modulus, combining the Young's Moduli $E_{i}$ and Poisson Ratios $\nu_{i}$ of the spheres. The mutual approach is defined as $\delta \equiv R_{1}+R_{2}-\left|\overrightarrow{r_{1}}-\overrightarrow{r_{2}}\right|$, where $\overrightarrow{r_{i}}$ is the position of a sphere's center of mass. The pressure at a radius $r$ is then completely defined by $a$ and $\delta$, and can be both positive (compressive) and negative (tensile). The largest compressive stress is found at $r=0$, and for now we will assume this stress does not reach the material yield strength, and thus plastic deformation does not occur.

While there is a singularity at the edge of the contact, the integral over the entire area is convergent and yields the (elastic) inter-particle force

$$
F_{\mathrm{E}}=\int_{0}^{a} 2 \pi r p(r) \mathrm{d} r=\frac{2 E^{*}}{3 R}\left(3 a \delta R-a^{3}\right) .
$$

The elastic strain energy stored in the contact equals [21]

$$
U_{\mathrm{E}}=\frac{E^{*} a^{3}}{3 R}\left[\delta\left(\frac{3 \delta R}{a^{2}}-1\right)-\frac{a^{2}}{5 R}\left(\frac{5 \delta R}{a^{2}}-3\right)\right] .
$$




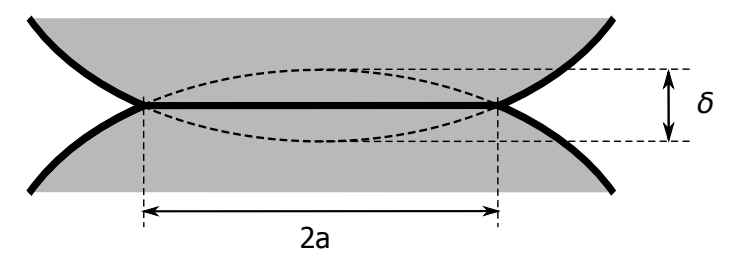

Figure 1: Schematic of the contact between two elastic spheres, showing the contact radius $a$ and mutual approach $\delta$.

So far we have deliberately not assumed any relation between $a$ and $\delta$. In some cases however, such relations do exist, and we will discuss two of these here, as we will compare them to the viscoelastic model later.

2.1.1. Hertz theory In the non-adhesive case, as described by [22], only repulsive forces are considered. For a given $\delta$, the corresponding equilibrium contact size can be found by minimizing $U_{\mathrm{E}}$, resulting in

$$
a^{2}=R \delta,
$$

which, together with (2) returns the famous Hertzian force

$$
F_{\mathrm{H}}=\frac{4 E^{*} a^{3}}{3 R} .
$$

From these two equations we see that in the non-adhesive case the force and contact size go to zero for $\delta=0$, and no solution exists for $\delta<0$. Additionally, the force is always positive, so no stable configuration of touching spheres is possible in the absence of external forces.

2.1.2. Johnson Kendall Roberts theory To describe the adhesive case, Johnson and coworkers [23] added a surface energy term

$$
U_{\mathrm{S}}=-\pi a^{2} \gamma
$$

where $\gamma$ is the surface energy. Thus, when the circular contact area shrinks or expands, the surface energy changes. For a certain value of $\delta$, the contact area will adjust itself so that

$$
\frac{\partial}{\partial a}\left(U_{\mathrm{E}}+U_{\mathrm{S}}\right)=0
$$

which can be solved to give

$$
\delta=\frac{a^{2}}{R}-\sqrt{2 \pi \gamma a / E^{*}}
$$

and consequently

$$
F_{\mathrm{JKR}}=\frac{4 E^{*} a^{3}}{3 R}-\sqrt{8 \pi \gamma E^{*} a^{3}}
$$

These final two relations describe Johnson Kendall Roberts theory (hereafter JKR theory), and show that contact between adhesive particles can be maintained even for 
negative approaches and negative forces. An important point is that an equilibrium exists at

$$
a_{\mathrm{eq}}=\left(\frac{9 \pi \gamma R^{2}}{2 E^{*}}\right)^{1 / 3}
$$

where the inter particle force equals zero, assuming no external forces are acting. Indeed, a pull-off force of $F_{c}=-(3 / 2) \pi \gamma R$, independent of elastic properties, has to be overcome in order to separate two spheres.

Several alternative adhesive theories exist. JKR theory is the result of assuming that no forces act outside of the contact area. Also in the seventies, Derjaguin and coworkers [24] took the opposite approach, by assuming the contact size is Hertzian, and adhesive forces to act in a ring around it. In 1977 it was shown by Tabor [25] that both theories are in fact limiting cases, whose validity depends on the value of the Tabor parameter, defined as

$$
\mu \equiv\left(\frac{R \gamma^{2}}{E^{* 2} z_{0}^{3}}\right)^{1 / 3}
$$

with $z_{0}=0.2 \sim 0.4 \mathrm{~nm}$ the spacing between atoms. For $\mu>5$, i.e. large compliant spheres, JKR theory is valid, while for $\mu<0.1$ Derjaguin Muller Toporov (DMT) theory is preferred. In the transition regime the Maugis-Dugdale solution can be used $[26,27,28]$.

\subsection{Dynamic contact}

Here we ask ourselves what happens when the material making up the spheres is not perfectly elastic. More specifically, the material will be assumed to be linearly viscoelastic, with a single relaxation time $T_{\text {vis }}$. We focus first on what this means for the contact edge, after which we turn our attention to the bulk of the material.

2.2.1. Viscoelastic crack theory The growing or receding contact area can be described as a Mode I crack of length $2 \pi a$ that is either closing or opening. For an infinite linear elastic material the crack is adequately described by Griffith theory [29], returning (6). For viscoelastic materials however, this approach breaks down as it predicts infinite strain rates for any non-zero crack velocity§. The basis for a more advanced theory comes from Barenblatt [31], who provided a solution for the infinite stresses. Based on this concept numerous theories for propagating viscoelastic cracks have been developed $[32,33,34,35,36,37]$. The main result of these studies is that energy is dissipated at

$\S$ This problem was circumvented by [30], who integrated over the elastic stresses before taking the timederivative (their equation 30). While the integral over the elastic stress is finite, and indeed equal to the elastic force, the integral over the time-derivative of the elastic stress does not converge. More specific, swapping the integral and derivative using Leibniz's rule results in a boundary term proportional to $\dot{a} p(a)$, which is infinite in JKR-theory. A more detailed theory of what happens close to the contact edge is needed, and has become available in the form of viscoelastic crack theory described here. 
the crack tip, creating an asymmetry between opening and closing cracks, which causes (7) to break down. However, providing

$$
\frac{\partial U_{\mathrm{S}}^{*}}{\partial a}=-2 \pi a G_{\mathrm{eff}}(\dot{a})
$$

is the energy released/absorbed when the crack is closed/opened, and includes any viscoelastic losses at the crack tip, we may write

$$
\frac{\partial}{\partial a}\left(U_{\mathrm{E}}+U_{\mathrm{S}}^{*}\right)=0
$$

In (12), the effective surface energy $G_{\text {eff }}$ is a function of crack velocity $\dot{a}$. For sufficiently slow cracks, $G_{\text {eff }}=\gamma$, and (13) reduces to (7) as expected. In Appendix A we show for which crack opening velocity this occurs. For larger crack speeds however, $G_{\text {eff }}>\gamma$ for opening cracks, and $G_{\text {eff }}<\gamma$ for healing ones. This causes so-called adhesion hysteresis at the contact edge, and has been experimentally verified [38]. Combining (12) and (13) yields

$$
G_{\text {eff }}(\dot{a})=\frac{E^{*}}{2 \pi a R^{2}}\left(a^{2}-\delta R\right)^{2}
$$

For a certain combination of $a$ and $\delta$, the above relation can be used to find the current effective surface energy, and thus $\dot{a}$. The exact shape of $G_{\text {eff }}(\dot{a})$ is quite complex. Here, we will use results of Greenwood [39], who combined the work of Barenblatt and Schapery with a Maugis-Dugdale potential around the crack tip. The material is described as a three-element solid, with a relaxation timescale $T_{\text {vis }}$ and a ratio of relaxed to instantaneous elastic modulus $k$. The effective surface energy is then written as

$$
G_{\text {eff }}(\dot{a}) \equiv \beta(\dot{a}) \gamma
$$

which can be inserted into (12). Analytical functions are provided relating $\dot{a}$ to $\beta$. For the majority of crack speeds, and almost independent of $k$, we can use [39]

$$
\frac{\sigma_{0}^{2} T_{\text {vis }}}{E^{*} \gamma} \dot{a}= \begin{cases}0.15\left[\beta \log \left(\frac{1-k}{1-\beta}\right)\right]^{-1} & \text { for } \dot{a}>0 \\ -0.24 \beta\left[\log \left(\frac{1-k}{1-1 / \beta}\right)\right]^{-1} & \text { for } \dot{a}<0\end{cases}
$$

where $\sigma_{0} \simeq \gamma / z_{0}$ is the size of the attractive force acting in the region described by the Maugis-Dugdale model. For low speeds, (16) looses accuracy, and [39] provides, specifically for $k=0.02$,

$$
\frac{\sigma_{0}^{2} T_{\text {vis }}}{E^{*} \gamma} \dot{a}= \begin{cases}(0.1035 x+0.3421) x^{1.116}, & \text { for } 0.29<\beta<1, \\ \text { where } x=(1 / \beta-1) & \\ -(0.2112 x+0.3939) x^{1.1403}, & \text { for } 1<\beta<3.7, \\ \text { where } x=(\beta-1) . & \end{cases}
$$

These relations have successfully been used to explain adhesion hysteresis in oscillatory normal loading [40], and in the rolling contact of polymers [41]. More recently, Barthel 
[42] studied viscoelastic cracks for the same three-element material model, but replaced the Maugis-Dugdale potential with a more realistic one, finding results similar to (16) and (17).

The above form of adhesion hysteresis is often called "mechanical hysteresis" [43], and disappears when the contact loading/unloading cycle is performed at infinitely low velocities. A different kind is "chemical hysteresis", where material close to the surface is left in a physically different state after the loading/unloading cycle [43, 44, 45]. In this work, we assume mechanical hysteresis to be the dominant process.

2.2.2. Bulk dissipative force When two viscoelastic spheres are pressed together at a finite velocity, a significant amount of energy might also be dissipated in the bulk of the material. When dissipation and strain rates are small everywhere, the total stress tensor can be written as a linear combination of the elastic and a dissipative stress tensor [46]. We follow this approach, and write the dissipative stress as being proportional to the time-derivative of the elastic one $[1,11]$. Realizing we are interested only in the normal component of the dissipative stress within the contact area, we can write

$$
\sigma_{\text {dis }}(r)=A \frac{d}{d t} p(r)=A\left[\frac{\partial p(r)}{\partial \delta} \dot{\delta}+\frac{\partial p(r)}{\partial a} \dot{a}\right],
$$

where $p(r)$ is the elastic pressure distribution given by (1), and the proportionality constant $A$ is a combination of viscous and elastic constants with units of time, which we approximate as $A \sim T_{\text {vis }} / \nu^{2}[1,30]$.

Similar to (2), the dissipative stresses can be integrated to yield the bulk dissipative force. The integration should be over all the dissipative stresses, except for those arising from the crack propagation, which are accounted for by the viscoelastic crack theory described in section 2.2.1. More specifically, we will separate the influence of the bulk

deformation from the crack-induced stresses by only taking into account the $\dot{\delta}$-term in (18). This allows us to write

$$
F_{\mathrm{D}}=A \int_{0}^{a} 2 \pi r \dot{\delta} \frac{\partial p(r)}{\partial \delta} \mathrm{d} r=2 A E^{*} a \dot{\delta} .
$$

The dissipative force thus depends on both $a$ and $\dot{\delta}$, becomes negligible when the contact size goes to zero, and always has the same sign as $\dot{\delta}$, ensuring it will act like a true drag term and always oppose motion.

\section{Head-on collisions}

The contact model put forward in section 2 can be used to calculate the evolution of the contact between two spheres that collide head-on. In this section, we limit ourselves to equal-sized spheres of the same material, but the theory described here can be applied to sphere-wall collisions by adjusting the effective mass and radius accordingly. The material properties are chosen to resemble water-ice microspheres (table 2). This combination of material properties has a Tabor parameter of $\mu \simeq 14$, which would put 

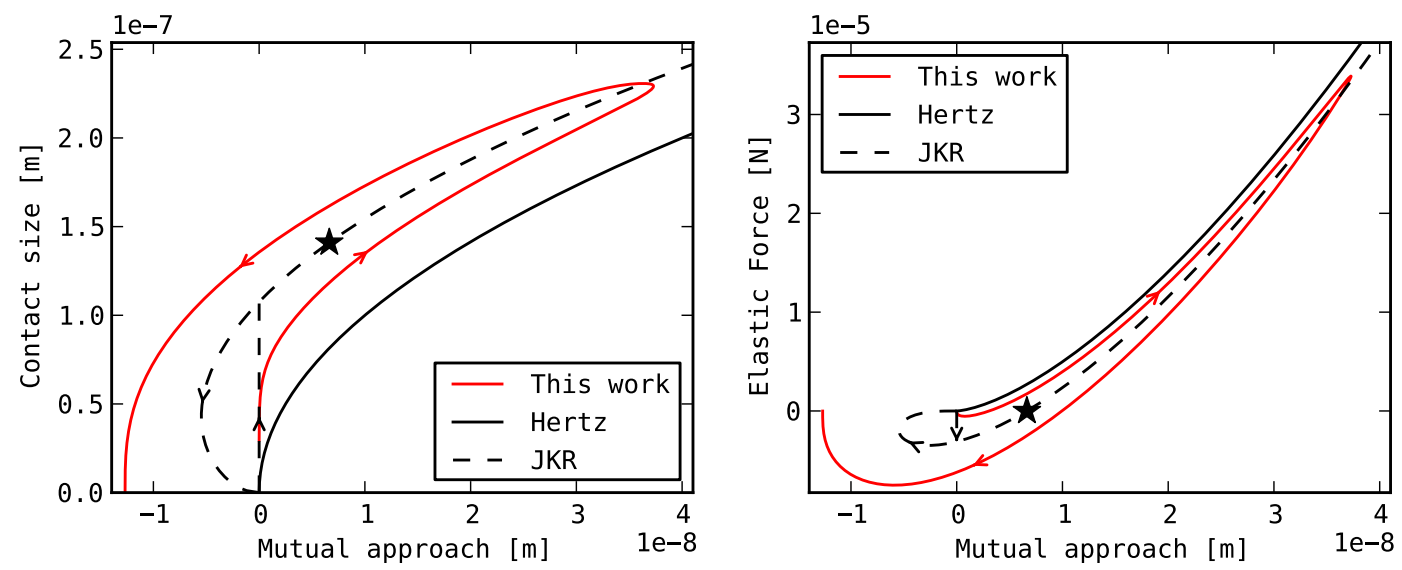

Figure 2: Evolution of the contact size (left) and elastic force (right) versus mutual approach, for an $8 \mathrm{~m} \mathrm{~s}^{-1}$ bouncing collision with the properties of ice (table 2) and a viscous relaxation time of $T_{\text {vis }}=10^{-11} \mathrm{~s}$. Predictions of Hertz $((4)$ and (5)) and JKR theory $((8)$ and $(9))$ are also plotted, and the star marks the equilibrium point in JKR theory. The vertical dashed lines show the snap-on in JKR theory, where the contact radius and force jump to a finite value when the spheres first touch at $\delta=0$.

this in the JKR limit when viscoelasticity was not present. The value of $T_{\text {vis }}$ is regarded as unknown, and will be varied later on to probe the effect the viscoelastic nature has on the evolution of the collision.

In section 2.2 we obtained the elastic and dissipative force between two spheres as a function of the contact size, their mutual approach, and the time-derivatives of these variables. These are all the tools we need to numerically integrate a collision at a certain velocity, realizing that the evolution of the mutual approach is given by

$$
\ddot{\delta}=-\frac{1}{m^{*}}\left(F_{\mathrm{E}}+F_{\mathrm{D}}\right),
$$

where the forces are given by (2) and (19). The reduced mass $m^{*-1}=m_{1}^{-1}+m_{2}^{-1}$ can be written in terms of density and radius. Meanwhile, the evolution of the contact size is governed by (14) - (17). The moment of first contact is taken as $t=0$, and as we do not allow for long-range forces the initial conditions for the mutual approach are

$$
\left\{\begin{array}{l}
\delta(0)=0, \\
\dot{\delta}(0)=v_{\text {in }} .
\end{array}\right.
$$

As (14) does not allow $a=0$, we have to make an analytical approximation for the initial growth of the contact area, see Appendix B. Using these initial conditions, (14), (16), (17) and (20) have to be solved simultaneously to obtain the evolution of $\delta$ and $a$ in time.

\subsection{Bouncing collision}

Figure 2 shows the evolution of the contact size and elastic force as a function of mutual approach, for a head-on collision at $8 \mathrm{~m} \mathrm{~s}^{-1}$, and $T_{\text {vis }}=10^{-11} \mathrm{~s}$. The relations from 
Hertz theory and JKR theory are also shown, and the star marks the equilibrium point in JKR theory $\left(F_{\mathrm{JKR}}=0\right)$. At the start of the collision, $\delta=0$, and it is clearly visible in the left panel of figure 2 that the contact area initially grows very fast, as its radius increases to $\sim 0.1 \mu \mathrm{m}$ with $\delta$ hardly changing. This "snapping on" is a consequence of the adhesive forces, and results in the contact being larger then predicted by Hertz theory. As a direct consequence, the force is negative early on, and the spheres are accelerated towards each other. However, the effective surface energy is smaller than the static value, so both the contact radius and the force do not reach the JKR-value. As the spheres compress, kinetic and surface energy are converted into elastic energy $\|$, and the spheres are brought to a halt at some maximum $\delta$. Here, the motion is reversed, and the spheres start to move apart. During unloading (rebound), the contact area is shrinking, and the effective surface energy is larger than the static one, causing the contact area to be larger than expected from JKR, and the force to be smaller. During the rebound, the spheres will cross the point $\delta=0$, but maintain contact as a result of the attractive surface forces. Because of the large effective surface energy, the maximum tensile force can be seen to be a factor 2 or so larger than the classic pull-off force from JKR theory, and contact can be maintained for even longer. Since both $a$ and $\delta$ are followed individually, the collision can be integrated as $a$ goes to zero. The total collision time, $\sim 1.7 \times 10^{-8} \mathrm{~s}$, can be compared to the one expected from Hertz theory, which can be estimated as $[9,12]$

$$
t_{\mathrm{H}}=2.87\left(\frac{m^{* 2}}{R E^{* 2} v_{\text {in }}}\right)^{1 / 5}
$$

and equals, for this particular set-up, $1.4 \times 10^{-8} \mathrm{~s}$. Thus, the combined effects of viscoelasticity and adhesion have lengthened the collision by about one fifth.

\subsection{Sticking collision}

At lower velocities, the pre-collision kinetic energy becomes comparable to the dissipated energy, and sticking can be achieved. Figure 3 shows the evolution of the contact for a collision similar to the one from figure 2 , but at a velocity of $v_{\text {in }}=2 \mathrm{~m} \mathrm{~s}^{-1}$. Initially, the collision resembles the faster one, but as there is less kinetic energy available, the maximum contact size and mutual approach reached are somewhat smaller. During rebound, the spheres are not able to separate, and instead oscillate back and forth, spiraling towards the JKR equilibrium point as a result of the dissipative effects. In a purely elastic scenario, the oscillation would not be dampened. The kinetic energy is dissipated within a couple of oscillations, on a timescale of a few times $10^{-8} \mathrm{~s}$ (see Appendix C). The energy absorbed as a result of the dissipative properties will be converted into heat. At this stage, we do not take into account any effects an increased temperature might have on the material properties, and simply view this energy as lost.

\|I In Appendix C the energy budget during the collision is described in more detail. 

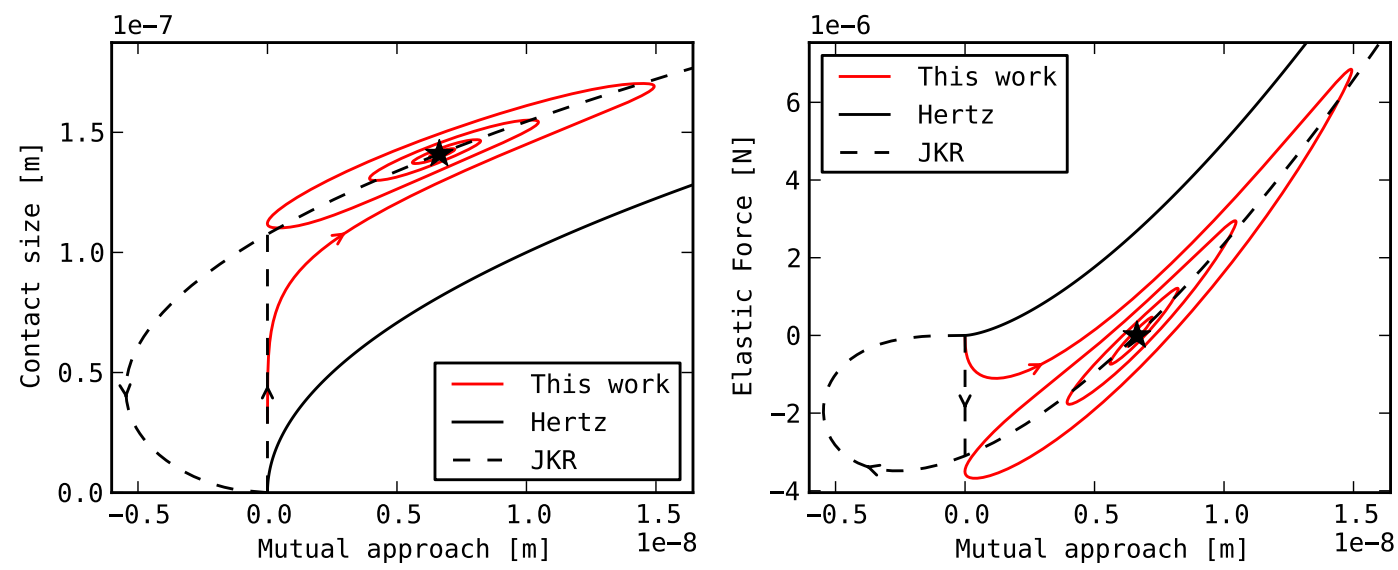

Figure 3: Evolution of the contact size (left) and elastic force (right) versus mutual approach, for a $2 \mathrm{~ms}^{-1}$ sticking collision with the properties of ice (table 2) and a viscous relaxation time of $T_{\mathrm{vis}}=10^{-11} \mathrm{~s}$. Predictions of Hertz ((4) and (5)) and JKR theory ((8) and (9)) are also plotted, and the star marks the equilibrium point in JKR theory. The vertical dashed lines show the snap-on in JKR theory, where the contact radius and force jump to a finite value when the spheres first touch at $\delta=0$.

\subsection{Coefficient of restitution}

To capture the outcome of a collision in a single quantity, we calculate the coefficient of restitution $e_{\mathrm{A}}$. There are several definitions for this coefficient in terms of velocity or energy [47], but for the normal collisions described in this work the two are identical, and we can write the coefficient of restitution as

$$
e_{\mathrm{A}}=-\frac{\dot{\delta}\left(t_{c}\right)}{\dot{\delta}(0)},
$$

where $t_{c}$ is the collision time. The resulting value can be compared to two cases in which analytical estimates are possible. In the elastic JKR limit the coefficient of restitution equals $[12,48]$

$$
e_{\mathrm{JKR}}=\sqrt{1-\left(\frac{v_{c}}{v_{\mathrm{in}}}\right)^{2}},
$$

where

$$
v_{c}=\left(\frac{14.18}{m^{*}}\right)^{1 / 2}\left(\frac{\gamma^{5} R^{4}}{E^{* 2}}\right)^{1 / 6},
$$

is the sticking velocity, below which all collisions will result in sticking. Alternatively, we may look at the non-adhesive viscoelastic case. In the limit where dissipation is small, the coefficient of restitution can be estimated as $[1,49]$

$$
e_{\mathrm{D}} \simeq 1-5.57\left(\frac{A}{t_{\mathrm{H}}}\right) \text {. }
$$




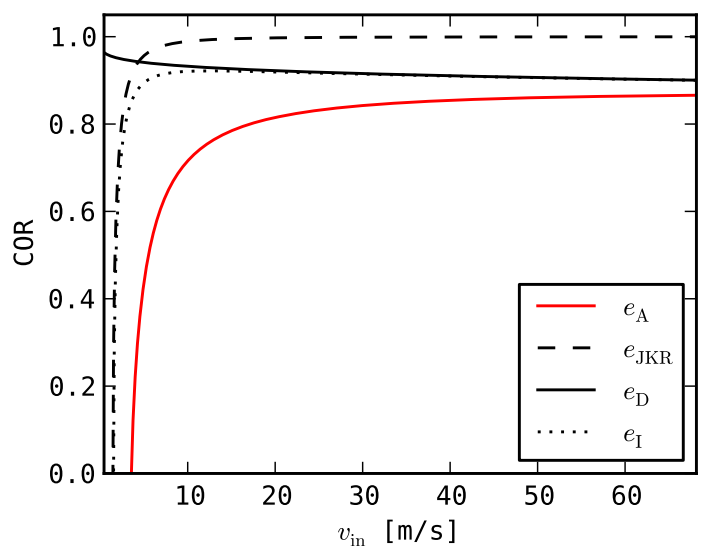

Figure 4: Coefficient of restitution (COR) for head-on collisions as a function of impact velocity (solid red). Properties of ice and a viscous relaxation time of $T_{\text {vis }}=10^{-11} \mathrm{~s}$ are used.

If one were to assume adhesion and viscoelastic dissipation do not influence each other, these two energy losses can be added to yield

$$
e_{\mathrm{I}}=\sqrt{e_{\mathrm{JKR}}^{2}+e_{\mathrm{D}}^{2}-1}
$$

However, adhesion and viscoelasticity cannot be treated independently as substantially more energy is dissipated. For example; the collision described in figure 2 has $e_{\mathrm{I}}=0.92$, while the numerical viscoelastic model employed here results in $e_{\mathrm{A}}=0.66$.

Figure 4 shows the variation of the coefficient of restitution with velocity, and compares it to the above estimates. Focussing first on the lowest velocities, we see that the sticking velocity is increased substantially. Towards the higher velocities, the results appear to approach the limit described by (26). Comparison with (27) shows that treating adhesion and viscoelasticity separately will significantly underestimate not only the sticking velocity, but the amount of energy dissipation over the entire velocity range. The effect of varying the reduced radius is shown in figure 5, where collisional outcomes at a fixed velocity are shown. Again, the difference between the theory developed here and (27) is substantial, although it vanishes for larger spheres, where the kinetic energy is much larger than the dissipated energy, and collisions are almost completely elastic.

An important parameter for many studies is the sticking threshold velocity $v_{s}$; the maximum velocity at which colliding particles will stick. Figure 5 shows the sticking velocity as a function of reduced radius and viscous relaxation time for water-ice particles. The sticking velocity in the non-viscous case (25) is plotted for comparison. For very small values of $T_{\text {vis }}$ the material effectively behaves elastically and (25) is retrieved. For larger values of the relaxation time, the sticking velocity can be increased significantly, especially for small particle sizes. 

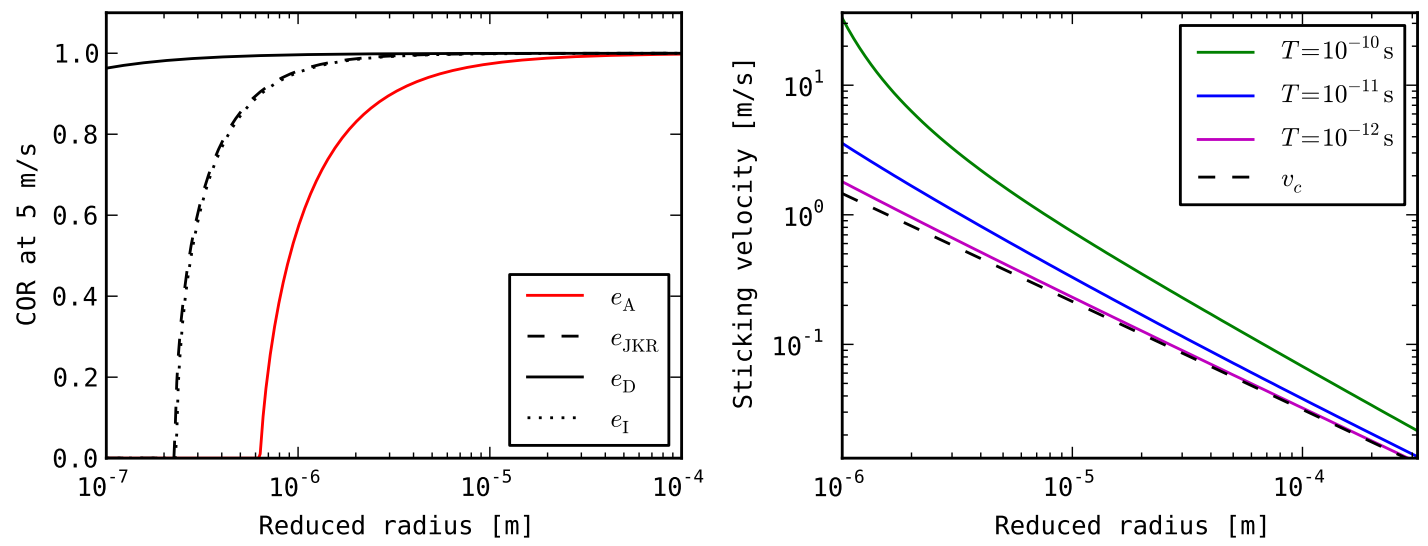

Figure 5: Left: Coefficient of restitution for head-on collisions as a function of reduced radius. The material properties of ice and a viscous relaxation time of $T_{\text {vis }}=10^{-11} \mathrm{~s}$ have been assumed. Right: Sticking velocity as a function of reduced radius for the material properties of ice. The non-viscous sticking velocity of (25) is plotted for comparison.

\subsection{Plastic deformation}

During a collision, the stress in the material can become so large that it starts to yield plastically. In a contact between spheres, ignoring adhesion and viscosity, the maximum shear stress is attained right beneath the contact area and on the axis of symmetry. Using either the Tresca or von Mises criterion, it can be shown that plastic deformation in the region below the contact will start when the pressure in the center of the contact area $p_{0}$ exceeds 1.6 times the yield strength $Y$ of the material in simple tension [12]. We can write a condition for the impact velocity for which yield is just initiated.

$$
v_{\mathrm{y}}^{2}=10.2 \frac{R^{3} p_{\mathrm{y}}^{5}}{m^{*} E^{* 4}}
$$

where $p_{\mathrm{y}}=1.6 Y$ denotes the pressure at the center of the contact area at the onset of plastic deformation [12]. Note that since $v_{\mathrm{y}}$ scales with $R^{3} / \mathrm{m}^{*}$, the yield velocity does not depend on size, assuming $Y$ is independent of size. When the impact velocity is larger than $v_{\mathrm{y}}$, a plastic region will be created underneath the contact area, and the pressure distribution will flatten off [50]. For even faster collisions the maximum pressure grows from $1.6 Y$ to about $3 Y$, at which point the plastic region reaches the sphere's free surface, plastic flow is no longer contained and the situation becomes more complicated [12].

An analytical model for the coefficient of restitution is provided by Thornton and Ning [48], where the pressure distribution is cut off above a critical $p_{\mathrm{y}}$. It is shown that this cutoff results in a force which depends linearly on the approach $\delta$. In addition, Thornton and Ning assumed that during the rebound phase the forces are Hertzian, but the radius of curvature is altered because of the plastic yield during loading. The 
resulting coefficient of restitution equals

$$
e_{\mathrm{P}}= \begin{cases}1 & \text { for } \hat{v}>1 \\ (6 \sqrt{3} / 5)^{1 / 2}\left(1-\frac{1}{6} \hat{v}^{2}\right)^{1 / 2} & \\ \times\left[\hat{v} /\left(\hat{v}+2 \sqrt{\frac{6}{5}-\frac{1}{5} \hat{v}^{2}}\right)\right]^{1 / 4} & \text { for } \hat{v} \leq 1\end{cases}
$$

where $\hat{v}=\left(v_{\mathrm{y}} / v_{\text {in }}\right)$. If no plastic deformation occurs, the collision is perfectly elastic and $e_{\mathrm{P}}=1$. If the impact velocity exceeds $v_{\mathrm{y}}$, the coefficient of restitution will drop off. At velocities well above the yield velocity $e_{\mathrm{P}} \propto\left(v_{\mathrm{y}} / v_{\text {in }}\right)^{1 / 4}$. More advanced models exist, that treat the variation of the central pressure and the unloading phase more carefully $[51,52]$ or include adhesion [53]. However, for the purpose of this work, we choose the model of Thornton \& Ning to describe the plastic behavior. For the constant cutoff pressure we use $p_{\mathrm{y}}=1.6 Y$.

For typical material parameters the yield velocity lies well above the sticking velocity [48], and it seems reasonable to assume the effects of adhesion and plastic deformation can be treated separately. Assuming the energy losses resulting from adhesion/viscosity and plastic deformation are being additive, we can write the total coefficient of restitution as

$$
e=\sqrt{e_{\mathrm{A}}^{2}+e_{\mathrm{P}}^{2}-1},
$$

where $e_{\mathrm{A}}$ is obtained by numerically integrating $\dot{\delta}$ in (23).

\section{Comparison to experiments}

The model presented in section 3 can be compared to experiments performed on colliding spheres where adhesion is of some importance, i.e. small spheres and/or low velocities. Experiments with colliding microspheres have been performed for some decades now, with results often in the form of a series of coefficients of restitution as a function of velocity. In general, most experimental results look similar to figure 4, and also show some dissipation towards higher velocities. In addition, fitting a purely elastic adhesive model has on some occasions yielded unrealistically high values for the surface energy [18]. In this section we set out to fit the model to a large set of published experimental results, with the aims of testing its applicability, and deriving the key parameters that govern the adhesive, viscoelastic, and plastic behavior.

\subsection{Method}

Available collision experiments reporting coefficients of restitution have been collected and reviewed by Güttler et al [19]. Here, we select those experiments from the dataset that resemble head-on and non-rotating collisions, and where the effects of surface 
roughness are believed to be small. Moreover, we are interested only in the data where a drop in the coefficient of restitution towards lower velocities is visible. With this, we hope to choose an unbiased sample, which is also as complete as possible to the best of our knowledge. The selected experiments are summarized in table 1 , where the materials, particle sizes, and examined velocities are shown. The micrometer experiments are all sphere-wall collisions, while the millimeter particles were collided sphere on sphere. Table 2 lists the various materials, together with their properties.

To fit the experimental results, we use the elastic material properties from table 2 , and treat $\gamma, T_{\text {vis }}$ and $Y$ as fitting parameters. While the surface energy might be considered as known, the values listed in table 2 under $\gamma_{\mathrm{L}}$ correspond to clean surfaces in perfect contact. These conditions are not necessarily met during an experiment, and the value of the surface energy might differ accordingly. Therefore, we allow $\gamma$ to vary and will compare the result to the literature value in section 4.2. For a particular experiment, the best fit is obtained by minimizing

$$
\chi^{2}=\frac{1}{N} \sum_{n=1}^{N}\left[e_{\exp }\left(v_{n}\right)-e\left(v_{n}\right)\right]^{2},
$$

where $N$ is the total number of data points, and the theoretical $e\left(v_{n}\right)$ is given by (30). The value of $\chi^{2}$ is calculated for a grid of values for $\gamma, T_{\text {vis }}$ and $Y$, after which the uncertainty in each individual parameter is taken as the range in which this parameter can vary without $\chi^{2}$ growing by more than a factor of 2 . For experiments where the error on the coefficient of restitution was provided, this method yielded uncertainties comparable to the size of the $1 \sigma$ confidence limit.

\subsection{Results}

We recognize three global characteristics in the experiments: (1) At low velocities, the outcome is dominated by adhesive and viscous forces, sometimes resulting in sticking. Just above the sticking velocity the COR rises steeply but does not reach unity. (2) Towards slightly higher velocities, the kinetic energy increases and the effects of adhesive forces decrease. However, as a result of the bulk dissipative force collisions are still dissipative, and regions where the COR is nearly constant and well below unity are observed for almost every experiment. These regions, covering up to factors of 3-4 in velocity, cannot be adequately explained by purely elastic adhesive theory, as the COR is expected to rise to 1 rapidly above the sticking velocity. (3) Some experiments show a drop in the COR towards high velocities. This drop is explained by plastic deformation. In the majority of the experiments however, the high velocities needed to observe this part are not reached.

Figure 6 shows two examples of the produced fits. All other fits, as well as a table giving the fitting parameters and uncertainties for each individual experiment, are available online as supplementary material. Perusal of the fits reveals good agreement between model and experiments. The three global characteristics outlined above are in a natural way contained with this theory in terms of the adhesion and the viscous 
Table 1: Summary of experiments used in this work. For a more detailed review of these experiments the reader is referred to [19].

\begin{tabular}{|c|c|c|c|c|}
\hline \multicolumn{5}{|c|}{ EXPERIMENTS WITH MICROMETER PARTICLES } \\
\hline Projectile material & target material & diameter $(\mu \mathrm{m})$ & velocity $\left(\mathrm{m} \mathrm{s}^{-1}\right)$ & Ref. \\
\hline polystyrene latex & quartz & 1.27 & $2-15$ & {$[13]$} \\
\hline polyvynil-toluene & quartz & 2.02 & $5-35$ & {$[13]$} \\
\hline ammonium fluorescein & mica & 4.9 & $5-120$ & {$[14]$} \\
\hline ammonium fluorescein & silicon & 4.9 & $5-110$ & {$[14]$} \\
\hline ammonium fluorescein & tedlar & 4.9 & $3-16$ & {$[14]$} \\
\hline ammonium fluorescein & molubdenum & 4.9 & $5-60$ & {$[14]$} \\
\hline Ag-coated glass & aluminium & 8.6 & $2-20$ & {$[15]$} \\
\hline Ag-coated glass & copper & 8.6 & $2-20$ & {$[15]$} \\
\hline Ag-coated glass & coated steel & 8.6 & $2-20$ & [15] \\
\hline Ag-coated glass & steel & 8.6 & $2-20$ & {$[15]$} \\
\hline Ag-coated glass & tedlar & 8.6 & $2-20$ & {$[15]$} \\
\hline steel & silicon & 55 & $0.4-2$ & {$[16]$} \\
\hline steel & silicon & 90 & $0.4-2$ & {$[16]$} \\
\hline Ag-coated glass & silicate & 40 & $0.04-0.44$ & {$[17]$} \\
\hline \multicolumn{5}{|c|}{ EXPERIMENTS WITH MILLIMETER PARTICLES } \\
\hline Projectile material & target material & diameter $(\mathrm{mm})$ & velocity $\left(\mathrm{m} \mathrm{s}^{-1}\right)$ & Ref. \\
\hline acrylic & acrylic & 3.96 & $0.1-1$ & {$[18]$} \\
\hline steel & steel & 3 & $0.05-0.5$ & [18] \\
\hline ceramics & ceramics & 3 & $0.05-0.5$ & [18] \\
\hline
\end{tabular}

Table 2: Different materials with their elastic properties and densities. The soundspeed has been calculated as $c_{s}=(E / \rho)^{1 / 2}$. Surface energy values come from $[20,54,55,56$, $57,58]$, and references therein.

\begin{tabular}{lccccl}
\hline Material & $E(\mathrm{GPa})$ & $\nu$ & $\rho\left(\mathrm{g} \mathrm{cm}^{-3}\right)$ & $c_{s}\left(\mathrm{~m} \mathrm{~s}^{-1}\right)$ & $\gamma_{\mathrm{L}}\left(\mathrm{J} \mathrm{m}^{-2}\right)$ \\
\hline aluminium alloy & 70 & 0.345 & 2.7 & 5092 & 0.59 \\
(stainless) steel & 190 & 0.28 & 7.92 & 4898 & 1.62 \\
molubdenum & 320 & 0.33 & 10.28 & 5579 & 2.81 \\
polished quartz & 54 & 0.17 & 2.6 & 4557 & 0.025 \\
silicon & 180 & 0.33 & 2.33 & 8789 & 1.42 \\
mica & 90 & 0.33 & 3. & 5477 & 0.77 \\
copper & 130 & 0.34 & 8.94 & 3813 & 1.11 \\
Ag-coated glass & 72 & 0.21 & 2.6 & 5261 & 0.61 \\
\hline \multicolumn{5}{c}{ POLYMERS } \\
\hline PSL & 3.4 & 0.33 & 1.026 & 1820 & 0.025 \\
tedlar & 2 & 0.33 & 1.46 & 1170 & 0.018 \\
acrylic & 3 & 0.35 & 1.22 & 1568 & 0.02 \\
lucite & 2.6 & 0.3 & 1.18 & 1484 & 0.02 \\
PVT & 3 & 0.33 & 1.2 & 1581 & 0.02 \\
AF & 1.17 & 0.33 & 1.35 & 931 & 0.01 \\
\hline \multicolumn{5}{c}{ OTHERS } \\
ceramics & 370 & 0.26 & 3.86 \\
water ice & 7 & 0.25 & 1.00 & 9790 & $0.04-5$ \\
\hline
\end{tabular}



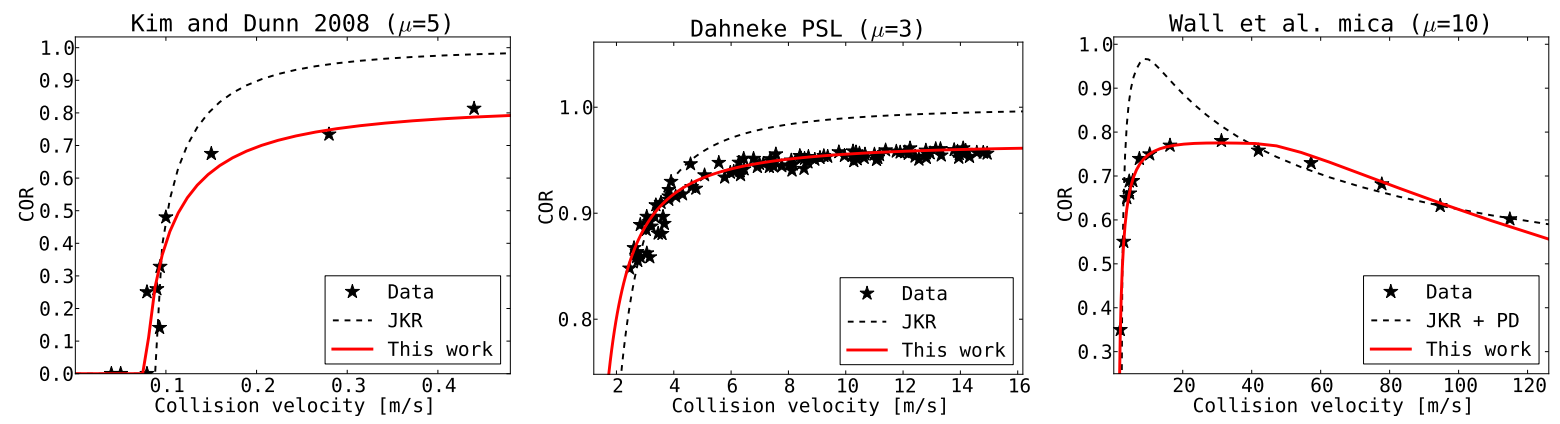

Figure 6: Three typical examples of the fits produced in section 4. Of these, only the rightmost experiment covers the plastic deformation regime. The dashed curves show JKR theory (24), where the surface energy has been chosen such that the low velocity part of the data is described well. For the rightmost curve, plastic deformation (29) was added when creating the dashed curve.

dissipation at the contact edge, the bulk viscous dissipation, and plastic deformation. For comparison, we plot in figure 6 curves neglecting viscoelasticity (dashed lines). It is clear that a model including only adhesive forces and plastic deformation is unable to describe the experimental results at intermediate velocities.

4.2.1. Surface energy Figure 7(a) shows the surface energy values for the best fitting models. The values have been normalized to the literature value, obtained by combining the corresponding surface energies from table 2 . The solid gray line indicates $\gamma=\gamma_{\mathrm{L}}$. The majority of the derived surface energies lie very close to the literature value.

A handful of experiments show a large uncertainty in the determination of $\gamma$, and we briefly discuss why. For Dahneke's PVT experiment, the reason is that the drop in the COR towards low velocities is not very distinct. For the mm-sized experiments the large uncertainty is a result of the scatter in the data, and the fact that the majority of the data points lie close to unity. As a result, model curves that combine a negligibly small value for $T_{\text {vis }}$ with a large surface energy, cannot be rejected on the basis of the method outlined above. However, non-zero values for the viscous relaxation time do describe the data more accurately, and the surface energies for these fits lies significantly closer to $\gamma_{\mathrm{L}}$, alleviating the issue of the high apparent surface energies as put forward by Sorace et al [18]. Moreover, measurements by Sorace et al of the COR at high speeds ${ }^{+}$range between 0.90 and 0.95 , supporting the hypothesis that the energy loss observed is caused by more than just adhesive forces.

4.2.2. Yield strength The obtained yield strengths are shown in figure 7(b), normalized to the Young's modulus of the softer material. The gray arrows correspond to

I For like materials, $\gamma$ equals twice the surface free energy, and for different materials the surface energies can be combined as $\gamma \simeq 2 \sqrt{\gamma_{1} \gamma_{2}}$.

+ These results are not included in the fitting procedure as it is unclear at which velocity they were obtained. 
(a) Surface energy

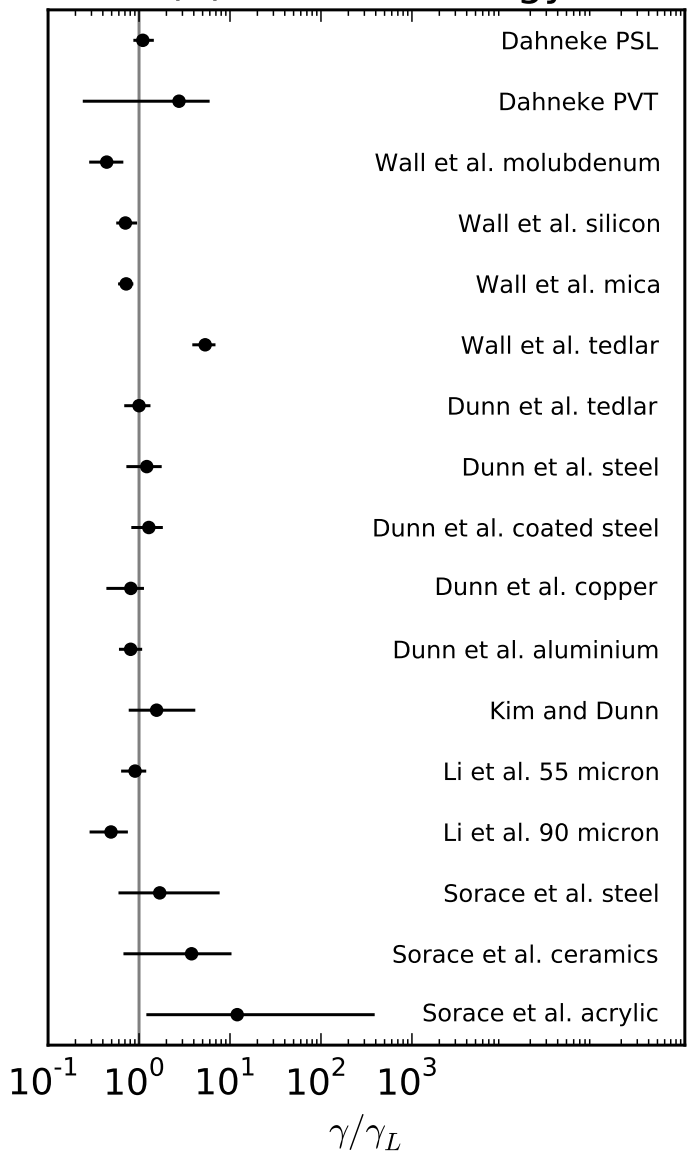

(b) Yield strength

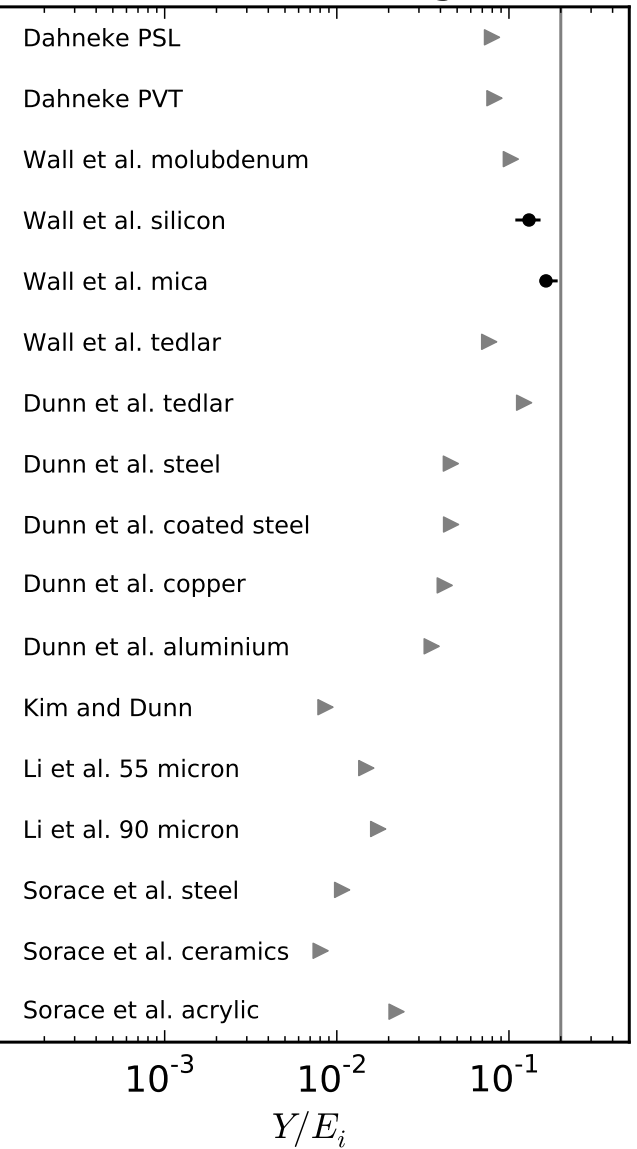

Figure 7: Best fit values for the surface energy and yield strength. The surface energy has been normalized to the literature value obtained from table 2, and the yield strength is normalized to the Youngs Modulus of the softer particle. The estimated error is indicated by the solid lines, and gray arrows indicate lower limits. Experiments are ordered from smallest (top) to largest (bottom) reduced radius.

experiments where plastic deformation does not occur at the velocities studied, and therefore give lower limits on the material strength*.

In macroscopic metal bodies plastic flow is the result of the motion of a dislocation through the crystalline lattice structure, and the yield strength is typically orders of magnitude smaller than the Young's modulus. The typical density of such dislocations that intersect a unit plane equals $10^{12}-10^{13} \mathrm{~m}^{-2}$, or between 1 and 10 per square micrometer [59]. On sub-micrometer scales, very few dislocations are available and the yield strength is expected to approach the theoretical strength of $0.2 E_{i}$, largely independent of material [60]. To describe the size dependence of the yield strength, an empirical power law of the form

$$
Y=Y_{0}+k l^{-n}
$$

* For the PVT [13] and tedlar and molubdenum experiments [14], the best fitting models did include plastic deformation towards high velocities, but models without could not be rejected. 
is often used, with $Y_{0}$ the macroscopic yield strength, $l$ the characteristic length scale, $k$ a constant, and $n=0.5$ the classical Hall-Petch value [61]. Experimentally derived values range between $0.5>n>1$ for metals $[62,63,64,65]$, suggesting there are multiple deformation mechanisms acting, that cannot be captured in a single power law [59]. For polymers a theoretical strength of $0.2 E_{i}$ has been predicted by Frenkel [66], while experimental values of the yield strength in bulk glassy polymers [67, 68] and metallic glasses [69] are about an order of magnitude smaller, and appear to be correlated with temperature as well as elastic modulus.

We estimate the length scale $l$ on which the plastic deformation occurs in the collision experiments, as being equal to the maximum Hertzian contact radius

$$
l=\left(\frac{15 m^{*} v_{\mathrm{in}}^{2} R^{2}}{16 E^{*}}\right)^{1 / 5} .
$$

To obtain a single value for $l$ for every experiment in table 1 , we use a representative velocity equal to half the maximum velocity used in that particular set up. The obtained normalized yield strengths are plotted in figure 8(a) as a function of $l$. For comparison, we plot the result of Lifshitz and Kolsky [70], who found a yield velocity of $0.3 \mathrm{~m} \mathrm{~s}^{-1}$ for steel spheres with a $3.18 \mathrm{~mm}$ radius. The observed material strengths vary by about a factor of 20, and appear to approach the theoretical strength for the smallest lengthscales. These results confirm that the drop in coefficient towards high velocities is indeed caused by plastic yield.

4.2.3. Relaxation timescale Of the fitted parameters, the viscous relaxation time is considered the most uncertain. Figure 8(b) shows the best fit values plotted against the reduced radius. For the theory of section 2 to be valid, the relaxation time needs to be small compared to the collision time $t_{c}$. As a lower limit for the collision time we can use the Hertzian approximation (22), realizing that any adhesive and/or viscous effects will act to increase the collision time. The Hertzian collision time is plotted as a gray solid line in figure $8(\mathrm{~b})$ for typical properties $\left(E^{*}=10 \mathrm{GPa}, \rho=2 \mathrm{~g} \mathrm{~cm}^{-3}\right.$ and $\left.v_{\text {in }}=10 \mathrm{~m} \mathrm{~s}^{-1}\right)$. The gray dotted line corresponds to $t_{\mathrm{H}} \times 10^{-3}$. The fitted values for the relaxation time lie well below the collision time, validating the use of our model. While the mm-sized experiments technically yield upper limits, we prefer to think viscoelastic behavior is important also at these sizes, as negligibly small values for $T_{\text {vis }}$ result in very large surface energies, and fail to explain the high-velocity points presented by Sorace et al [18]. The apparent relation between the relaxation time and reduced radius is curious, and will be further discussed in section 5 .

\section{Discussion}

A collision between spherical particles results in some kinetic energy being lost. As a result, the rebound velocity is smaller than the incoming velocity. In this work we put forward a collision model that treats adhesion and viscoelasticity in a self-consistent way, and assume the energy losses arising from plastic deformation to be additive. 
(a)

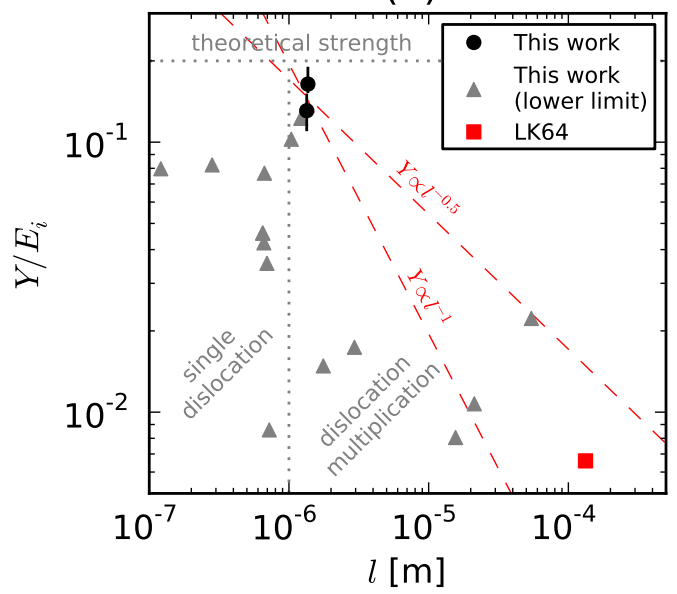

(b)

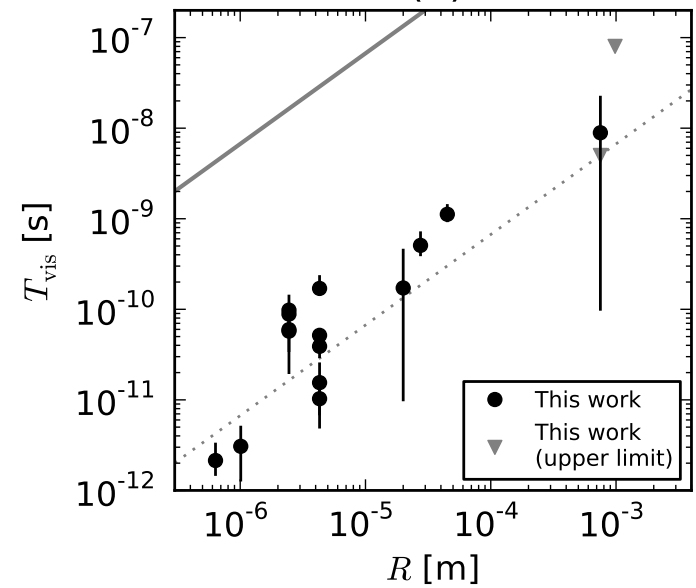

Figure 8: (a) Variation of normalized yield strength with particle radius. Gray triangles represent lower limits, and the horizontal dotted line shows the theoretical strength of $Y / E_{i}=0.2$. For $l>10^{-6} \mathrm{~m}$ many dislocations will be available, while they are scarce for smaller sizes (see text). The red square corresponds to data from [70]. The red dashed lines show $Y \propto l^{-0.5}$ and $Y \propto l^{-1}$ [59]. (b) Relaxation times as a function of reduced radius. The solid and dotted gray lines indicate typical values for $t_{\mathrm{H}}$ and $t_{\mathrm{H}} \times 10^{-3}$ respectively.

Incorporating dissipative viscoelastic stresses into a static contact description such as JKR theory is not straightforward. The problem is the existence of a theoretically infinite tensile stress at the contact edge, which causes infinite stress rates when the contact area grows or shrinks at a finite rate. Integration of the dissipative stresses will then inevitably result in an infinitely large dissipative force. A solution to this problem comes from viscoelastic crack theory [39], and is included in the contact description by allowing for an effective surface energy, which varies as a function of the rate with which the contact advances/recedes. To be able to describe the contact hysteresis that ensues, we follow the evolution in time of both the contact radius and the mutual approach. Some distance from the contact edge, the stress rates are dominated by the changes in the mutual approach, rather than the crack opening or closing. The dissipative stresses arising here can be integrated to yield a dissipative stress. With analytic expressions for the total normal force between two non-rotating particles, a head-on collision at a certain velocity can be solved numerically, yielding the total energy loss and coefficient of restitution.

Regarding the particle size and elastic modulus as known, the model is fitted to experimental results and yields values for the surface energy, the viscous relaxation timescale, and the yield strength. While the obtained values for the surface energy and yield strength compare well to expected values, it is difficult to judge whether the inferred material relaxation time is realistic, partly because of the viscoelastic model that is used, in which the material relaxes linearly on a single timescale. This is a 
very simplistic view, as more realistic materials contain numerous characteristic timeand length scales corresponding to (parts of) molecules, chains of molecules, or lattice structures. The experiments clearly reveal the effect of viscous dissipation for all particle sizes and therefore, by necessity, at all collision timescales. This immediately implies that the relevant viscous dissipation timescale is longer for collisions between larger grains (figure $8(\mathrm{~b})$ ). We interpret this along the lines outlined above: when larger volumes are involved in the collision process, larger structures can be activated in the dissipation process, and these larger structures by necessity require longer timescales to act.

The collision model used throughout this work does not take into account energy lost in the excitation of elastic waves, or the presence of surface asperities. We shall discuss these assumptions briefly. During a collision of two bodies, elastic waves will be excited that travel back and forth and carry a part of the initial kinetic energy. In the non-adhesive case this energy is expected to be negligible if the collision velocity is well below the material soundspeed [9], which is the case for the experiments encountered in this work (see table 2). However, the presence of adhesion might enhance the elastic wave energy around the lower velocities, increasing the sticking velocity slightly [20].

The presence of surface asperities can influence the collisional outcome profoundly. Irregularities that are large compared to the contact size will alter the local reduced radius, while smaller asperities can be crushed to dissipate energy [71] or survive and act to reduce the effective surface area and therefore the effect of adhesive forces [25]. Several authors provided information on the particle roughness. For the fluorescein particles, Wall et al [14] provided an upper limit on the roughness of $3 \mathrm{~nm}$. The steel particles were stated to be smooth to within the resolution of the scanning electron microscope (SEM), while the surface of the target showed a height standard deviation of $\sim 1 \mathrm{~nm}[16]$. An SEM was also used by Dunn et al [15] to study their surfaces, who found the steel to be virtually flawless within the resolution of $4 \mathrm{~nm}$. The softer targets showed some irregularities on the micron scale. Unfortunately, Dahneke [13] and Sorace et al [18] provided no information on the roughness of the particles they used. For more details on the surface conditions in the various experiments the reader is referred to Güttler et al [19] or the original experimental works. The typical diameter of the contact area ranges between about $100 \mathrm{~nm}$ and $20 \mu \mathrm{m}$ for the smallest and largest spheres described here, while the mutual approach is a factor $(a / R)$ smaller. Thus, contact will be made over many small asperities simultaneously, and their presence is not expected to significantly alter the collisional outcome. The obtained surface energies, as plotted in figure 7 , show no systematic deviation from literature values. Therefore, we believe that in general the effects of both elastic wave excitation and surface asperities are small. The collision model also assumes a Tabor parameter $\mu>5[25,28]$. The values of the Tabor parameter (calculated using $\gamma_{\mathrm{L}}$ ) are given in figure 6 and in the supplementary material. While two experiments are perhaps limiting cases, the majority of the experiments have $\mu \gg 5$, justifying the use of the collision model. 


\section{Conclusions}

In this work we have studied the collision between adhesive, viscoelastic spheres in a self-consistent way, by following the evolution of both the contact size, and the mutual approach of the sphere's centers of mass independently. The contact edge is described using viscoelastic crack theory, whereas deformations in the bulk of the material give rise to a dissipative force. The main results are:

- The combined effects of viscoelasticity in the bulk and at the contact edge can cause significant deviations from static adhesive contact theory. As a result, there is no unique relation between the contact size and the mutual approach.

- Because energy is dissipated through viscoelastic effects both in the bulk and at the contact edge, the sticking velocity can be significantly increased from the value predicted based on elastic adhesive theory. Well above the sticking velocity the viscoelastic effects still cause significant energy loss, and a coefficient of restitution of less then unity.

- Assuming viscoelasticity and adhesion can be treated separately results in a significant underestimation of the energy lost during a collision.

The model is extended to include plastic deformation at high velocities, and compared to a large set of experimental results resulting in the determination of the surface energy, material yield strength, and viscous relaxation time. The main findings can be summarized as follows:

- The collision model is remarkably successful in describing a large number of experiments, varying in experimental set-up, particle materials, particle size, and collision velocity. Removing any one of the three energy-loss mechanisms results in a dramatic decrease in the quality of the fit.

- The surface energy values obtained from the experiments are in good agreement with literature values. The majority of the derived surface energies lies within a factor of two of the expected values. The remaining small differences are expected and can easily be attributed to elastic waves and surface roughness.

- Through fitting the yield velocity, a value for the material yield strength can be obtained. For the majority of experiments plastic yield is not observed, resulting in a lower limit for this parameter. The resulting values for the yield strength lie within the expected range, and approach the theoretical strength of about $0.2 \times$ Young's modulus for the smallest sizes.

- When viscous dissipation is important, the relaxation timescale has to be well below the collision timescale. We observe a correlation between the relaxation timescale and the reduced radius of the sphere(s). We suggest that this relationship reflects the activation of relaxation in larger and larger structures as the collision size scale increases. 


\section{Acknowledgments}

Dust studies at Leiden Observatory are supported through the Spinoza Premie of the Dutch science agency, NWO. C.G. is grateful for the support of the Japan Society for the Promotion of Science (JSPS). D.H. acknowledges funding from the German Research Council (DFG) under grant B1298/11-1. The authors would like to thank J. Blum for encouraging discussions.

\section{Appendix A. Quasi-static limit}

Here we estimate the timescales below which the effects of viscosity are small, and the elastic limit is retrieved. Turning our attention first to the bulk dissipation, the effect of $T_{\text {vis }}$ becomes negligible when

$$
\frac{F_{\mathrm{D}}}{F_{\mathrm{E}}}<10^{-2}
$$

Plugging in (2) and (19), and assuming the Hertzian relation (4) reduces the above equation to

$$
T_{\text {vis }}<10^{-2} \nu^{2} t_{c}
$$

where we have used $(\delta / \dot{\delta}) \sim t_{c}$. Thus, bulk dissipation will have little effect on the energy balance if the relaxation timescale is much smaller than the collision timescale. A similar result can be obtained directly from (26).

The magnitude of the adhesion hysteresis depends on the crack velocity. We assume the quasi-static limit to be retrieved when the difference between effective and static

surface energy is of order $10^{-2}$. Making use of (17), appropriate for low crack velocities, we find this holds when

$$
\left(\frac{\sigma_{0}^{2} T_{\text {vis }}}{E^{*} \gamma}\right)|\dot{a}|<10^{-3} \text {. }
$$

In a collision, $\dot{a}$ will vary significantly, but we may obtain a typical value from Hertzian theory, where $\dot{a}=\dot{\delta}(R / 2 a)$, allowing us to rewrite the above limit as

$$
T_{\mathrm{vis}}<10^{-3} \frac{z_{0}}{a}\left(\frac{2 E^{*} z_{0}}{\gamma}\right) t_{c}
$$

Relaxation timescales longer than this will result in adhesion hysteresis, and affect the collisional outcome if the energy that is dissipated in this way is comparable to the kinetic energy. The fraction $E^{*} z_{0} / \gamma$ is relatively small for materials like ice or polymers, but can be substantially larger for metals or minerals like quartz (see table 2). As a result, the relative importance of crack and and bulk dissipation will vary per material.

For the ice particles considered in section 3, the term in brackets is of order unity, while $\left(z_{0} / a\right) \sim 10^{-3}$. Comparison of (A.2) and (A.4) reveals energy dissipation at the contact edge can take place for shorter timescales, and when the bulk of the material behaves elastically. For a $4 \mathrm{~m} \mathrm{~s}^{-1}$ collision between the ice spheres of table 2, (A.2) and (A.4) both hold for $T_{\text {vis }}=10^{-14} \mathrm{~s}$. For this value of the relaxation time, numerical 
integration of the equations of motion yields $e=0.93$, which is in excellent agreement with the quasi-static result: $e_{\mathrm{JKR}}=0.93$.

\section{Appendix B. Intial condition for contact area}

At the point of first contact, $a=\delta=0$, causing $G_{\text {eff }}$, as defined by (14), to be indefinite. To account for this, we take an approach very similar to section 4.1 of [72], the main difference being that our approximation is focussed on $\delta=0$, whereas the former uses $F_{\mathrm{E}}=0$.

Setting the mutual approach to zero in (14) returns

$$
a=\left(\frac{2 \pi R^{2} G_{\mathrm{eff}}}{E^{*}}\right)^{1 / 3} .
$$

As $\dot{a}$ is practically infinite, we can assume $G_{\text {eff }}$ should have the minimum value of $k \gamma$. In this case $(\beta=k)$, (16) cannot be used, and an analytical approximation is required. Following a similar approach as [72], we can approximate the time since contact formation, for a given $\beta$, as

$$
\begin{aligned}
1.198 T_{\text {vis }} \sigma_{0}^{2}\left(\frac{R}{E^{* 2} \gamma}\right)^{2 / 3} t & \simeq\left(s^{4}-1\right) \log \left(\frac{1-k}{1-s^{4}}\right) \\
& -1.5 \log \left(1+s+s^{2}\right) \\
& -\sqrt{3} \arctan \left(\frac{2 s+1}{\sqrt{3}}\right)+3 s\left(1+s^{3} / 4\right),
\end{aligned}
$$

where $s \equiv \beta^{1 / 3}$. Now, a value of $\beta$ arbitrarily close to $k$ can be chosen, and the above two equations will return the corresponding contact size, and time difference, providing a starting point for $\dot{a}$. The assumption that $\delta=0$ is justified as the contact initially grows so rapidly, the mutual approach hardly changes. For our numerical integrations, we have used the starting value of $G_{\text {eff }}=1.01 k \beta$.

\section{Appendix C. Energy budget during collision}

It proves useful to examine the various energies in the system during the collision. First, the kinetic energy is taken to equal

$$
U_{\mathrm{K}}=\frac{1}{2} m^{*} \dot{\delta}^{2}
$$

We already have an expression for the elastic energy in (3), and the surface energy is given by (6). The total amount of energy that has been dissipated at the crack tip at a time $t^{\prime}$ can be written as

$$
U_{\mathrm{CD}}=\int_{0}^{t^{\prime}} 2 \pi a(1-\beta) \gamma \dot{a} \mathrm{~d} t .
$$

This quantity is always positive, as $\dot{a}$ has the same sign as $(1-\beta)$. Lastly, the energy that has been dissipated in the bulk by $F_{\mathrm{D}}$ at some time $t^{\prime}$ is obtained through

$$
U_{\mathrm{BD}}=\int_{0}^{t^{\prime}} F_{\mathrm{D}} \dot{\delta} \mathrm{d} t .
$$




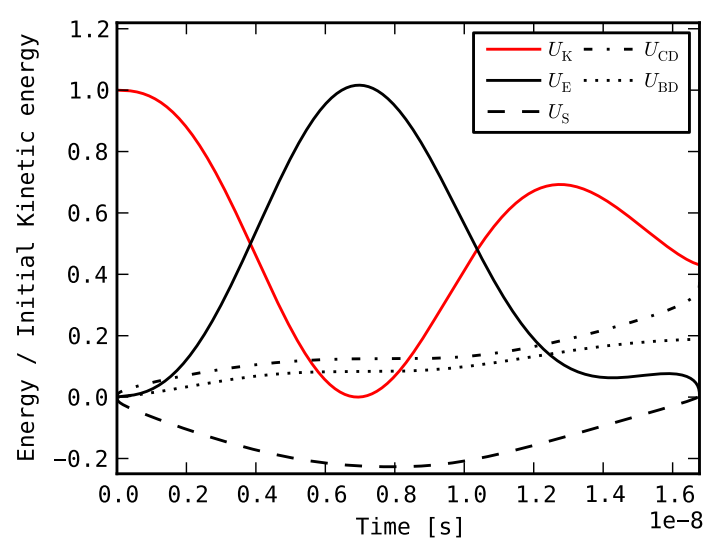

Figure C1: Evolution of the kinetic energy $\left(U_{\mathrm{K}}\right)$, elastic energy $\left(U_{\mathrm{E}}\right)$, surface energy $\left(U_{\mathrm{S}}\right)$, and energy dissipated at the crack $\left(U_{\mathrm{CD}}\right)$ and in the bulk $\left(U_{\mathrm{BD}}\right)$, for a $8 \mathrm{~m} \mathrm{~s}^{-1}$ bouncing collision with the ice properties of table 2 and a viscous relaxation time of $T_{\text {vis }}=10^{-11} \mathrm{~s}$. The energies are normalized to the initial kinetic energy of the collision.

Figure $\mathrm{C} 1$ shows these energies, normalized to the kinetic energy at $t=0$, for the same collision as described in figure 1 . The brief acceleration phase the spheres go through immediately after $t=0$ can be seen to have a negligible effect on the kinetic energy in this particular case. During the loading phase, the growth of the contact area results in energy being added to the system, and the surface energy grows increasingly negative. Energy is steadily dissipated in the bulk and at the crack tip. As the motion is reversed, the elastic energy starts to decrease while the surface energy grows, as new surface is being created. At the end of the collision, 37\% and 19\% of the initial kinetic energy has been dissipated at the crack tip and in de bulk of the material respectively, indicating that the adhesion hysteresis has a large influence on the energy balance in the system. Furthermore, the collision is clearly asymmetric, as the rebounding phase, which starts after $U_{\mathrm{K}}$ reaches zero, is significantly longer than the loading phase.

Figure $\mathrm{C} 2$ shows the various energies for the sticking collision of figure 4. Most of the initial kinetic energy and the released surface energy are dissipated (mainly at the crack tip), while a fraction remains stored in elastic energy. 


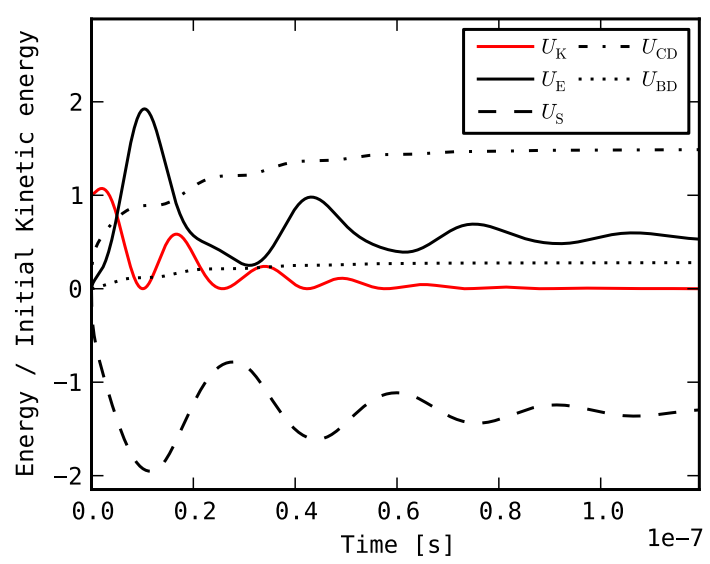

Figure C2: Evolution of kinetic energy $\left(U_{\mathrm{K}}\right)$, elastic energy $\left(U_{\mathrm{E}}\right)$, surface energy $\left(U_{\mathrm{S}}\right)$, and energy dissipated at the crack $\left(U_{\mathrm{CD}}\right)$ and in the bulk $\left(U_{\mathrm{BD}}\right)$, for a $2 \mathrm{~m} \mathrm{~s}^{-1}$ sticking collision with the ice properties of table 2 and a viscous relaxation time of $T_{\text {vis }}=10^{-11} \mathrm{~s}$.

\section{References}

[1] Brilliantov N V, Spahn F, Hertzsch J-M and Pöschel T 1996 Phys. Rev. E 53 5382-92

[2] Pöschel T, Brilliantov N and Schwager T 2003 Phys. A 325 274-83

[3] Ossenkopf V 1993 Astron. Astrophys. 280 617-46

[4] Dominik C and Tielens A G G M 1997 Astrophys. J. 480647

[5] Paszun D and Dominik C 2008 Astron. Astrophys. 484 859-63

[6] Wada K, Tanaka H, Suyama T, Kimura H and Yamamoto T 2009 Astrophys. J. 702 1490-501

[7] Salo H 1995 Icarus 117 287-312

[8] Salo H, Schmidt J and Spahn F 2001 Icarus 153 295-315

[9] Lord Rayleigh 1906 Philos. Mag. Series 611 283-91

[10] Reed J 1985 J. Phys. D: Appl. Phys. 182329

[11] Kuwabara K and Kono K 1987 Japan. J. Appl. Phys. 26 1230-3

[12] Johnson K L 1987 Contact Mechanics (Cambridge: Cambridge University Press)

[13] Dahneke B 1975 J. Colloid Interface Sci. 51 58-65

[14] Wall S, John W, Wang H-C and Goren S L 1990 Aerosol Sci. Technol. 12 926-46

[15] Dunn P F, Brach R M and Caylor M J 1995 Aerosol Sci. Technol. 23 80-95

[16] Li X, Dunn P F and Brach R M 1999 J. Aerosol Sci 30 439-49

[17] Kim O V and Dunn P F 2007 J. Aerosol Sci 39 373-5

[18] Sorace C M, Louge M Y, Crozier M D and Law V H C 2009Mech. Res. Commun. 36 364-68

[19] Güttler C, Heißelmann D, Blum J and Krijt S 2012 Normal Collisions of Spheres: A Literature Survey on Available Experiments (Preprint arXiv:1204.0001)

[20] Chokshi A, Tielens A G G M and Hollenbach D 1993 Astrophys. J. 407 806-19

[21] Muller V M, Yushchenko V S and Derjaguin B V 1980 J. Colloid Interface Sci. 77 91-101

[22] Hertz H 1882 J. Reine Angew. Math. 92 156-71

[23] Johnson K L, Kendall K L and Roberts A D 1971 Proc. R. Soc. A 324 301-13

[24] Derjaguin B V, Muller V M and Toporov Y P 1975 J. Colloid Interface Sci. 53 314-26

[25] Tabor D 1977 J. Colloid Interface Sci. 58 2-13

[26] Dugdale D 1960 J. Mech. Phys. Solids 8 101-4

[27] Maugis D 1992 J. Colloid Interface Sci. 150 243-69

[28] Johnson K L and Greenwood J A 1997 J. Colloid Interface Sci. 192 326-33

[29] Griffith A A 1921 Philos. Trans. Roy. Soc. London Ser. A 221 163-98

[30] Billiantov N V, Albers N, Spahn F and Pöschel T 2007 Phys. Rev. E 76 051302-12 
[31] Barenblat G I 1962 Adv. Appl. Mech. 7 55-129

[32] Schapery R A 1975 Int. J. Fracture 11 141-59

[33] Schapery R A 1975 Int. J. Fracture 11 369-88

[34] Greenwood J A and Johnson K L 1981 Phil. Mag. A 43 697-711

[35] Schapery R A 1989 Int. J. Fracture 39 163-89

[36] Hui C-Y, Baney J M and Kramer E J 1998 Langmuir 14 6570-8

[37] Baney J M and Hui C-Y 1999 J. Appl. Phys. 86 4232-41

[38] Maugis D and Barquins M 1978 J. Phys. D: Appl. Phys. 111989

[39] Greenwood J A 2004 J. Phys. D: Appl. Phys. 372557

[40] Wahl K J, Asif S A S, Greenwood J A and Johnson K L 2006 J. Colloid Interface Sci. 296 178-88

[41] Greenwood J A, Johnson K L, Choi S-H and Chaudhury M K 2009 J. Phys. D: Appl. Phys. 42 035301

[42] Barthel E and Frétigny C 2009 J. Phys. D: Appl. Phys. 42195302

[43] Chen Y L, Helm C A and Israelachvili J N 1991 J. Phys. Chem. 95 10736-47

[44] Chaudhury M K and Whitesides G M 1991 Langmuir 7 1013-1025

[45] Chaudhury M K and Owen M J 1993 J. Phys. Chem. 97 5722-6

[46] Landau L D and Lifshitz E M 1965 Elastizitätstheorie (Berlin: Akademie-Verlag)

[47] Stronge W J 1990 Proc. R. Soc. A 431 169-81

[48] Thornton C and Ning Z 1998 Powder Technol. 99 154-62

[49] Schwager T and Pöschel T 1998 Phys. Rev. E 57 650-4

[50] Hardy C, Baronet C N and Tordion G V 1971 Int. J. Numer. Meth. Eng. 3 451-62

[51] Wu C-Y, Li L-Y and Thornton C 2003 Int. J. Impact Eng. 28 929-46

[52] Wu C-Y, Li L-Y and Thornton C 2005 Int. J. Impact Eng. 32 593-604

[53] Mesarovic D J and Fleck N A 2000 Int. J. Solids Struct. 37 7071-79

[54] Gilman J J 1960 J. Appl. Phys. 31 2208-18

[55] Mark J E 1999 Polymer Data Handbook (Oxford: Oxford University Press)

[56] Siegel D J, Hector L G and Adams J B 2003 Phys. Rev. B 67092105

[57] Król P and Król B 2006 J. Eur. Ceram. Soc. 26 2241-8

[58] Kim O V and Dunn P F 2007 J. Aerosol Sci 38 532-49

[59] Kraft O, Gruber P A, Mönig R and Weygand D 2010 Annu. Rev. Mater. Res. 40 293-317

[60] Petch N J 1953 J. Iron Steel Inst. 17425

[61] Petch N J 1968 In Fracture ed Liebowitz H (New York: Academic Press)

[62] Brenner S S 1956 J. Appl. Phys. 27 1484-91

[63] Brenner S S 1957 J. Appl. Phys. 28 1023-6

[64] Volkert C A and Lelleodden E T 2006 Phil. Mag. 86 5567-79

[65] Zhu Y, Qin Q, Xu F, Fan F, Ding Y, Zhang T, Whiley B J and Wang Z L 2012 Phys. Rev. B 85 045443

[66] Frenkel J 1926 Z. Phys. A: Hadrons Nucl. 37 572-609

[67] Brown N 1971 Mater. Sci. Eng. 8 69-73

[68] Kozey V V and Kumar S 1994 J. Mater. Res. 9 2717-26

[69] Johnson W L and Samwer W 2005 Phys. Rev. Lett. 95195501

[70] Lifshitz J M and Kolsky H 1964 J. Mech. Phys. Solids 12 35-43

[71] Tsai C-J, Pui D Y H and Liu B Y H 1990 Aerosol Sci. Technol. 12 497-507

[72] Greenwood J A and Johnson K L 2006 J. Colloid Interface Sci. 296 284-91

\section{Online material}



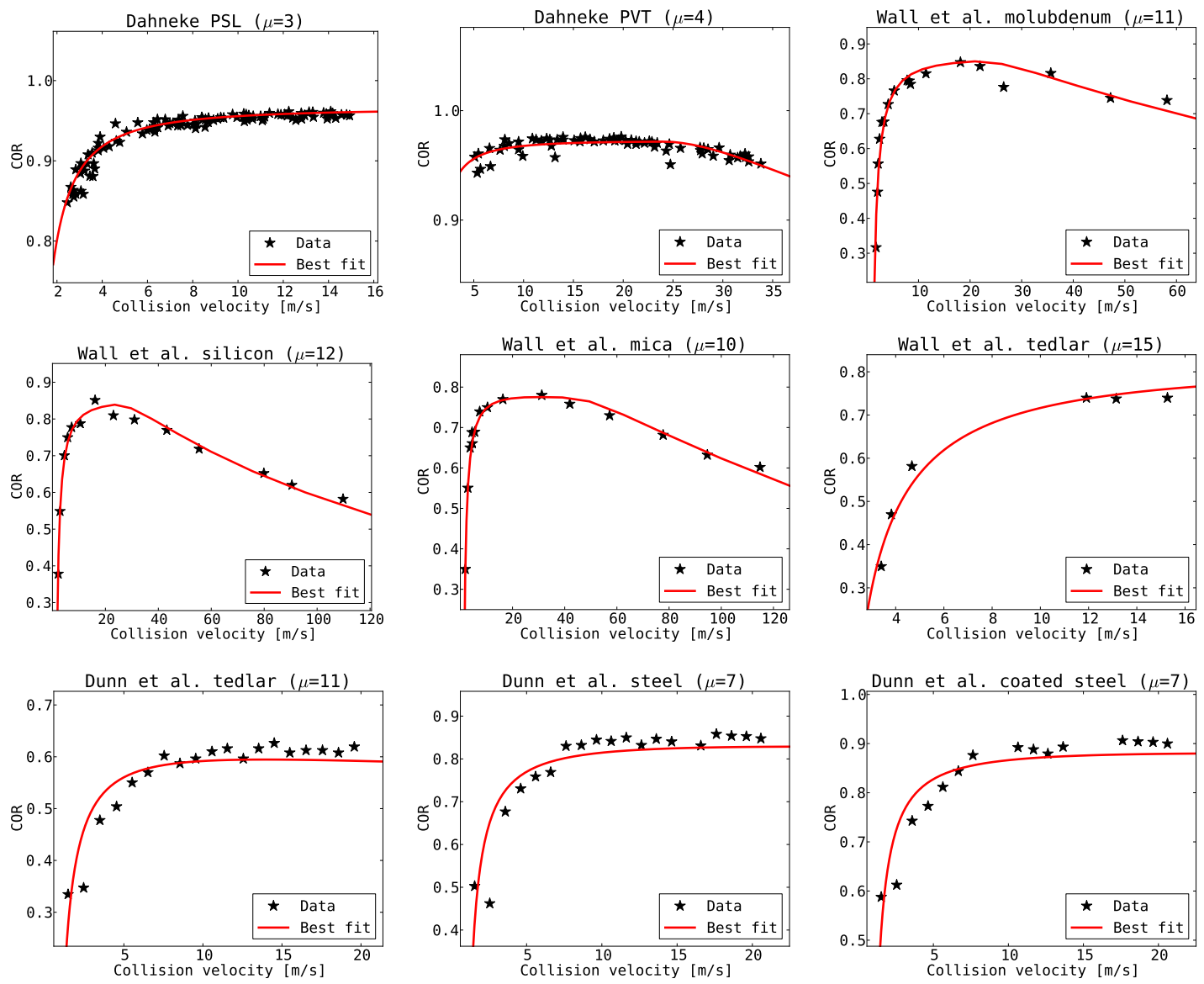

Figure C3: Best fitting models for the complete set of experiments. Note that the scale on the vertical axis varies. The value of the Tabor parameter $\mu$ (section 5 ) is shown above each plot. 

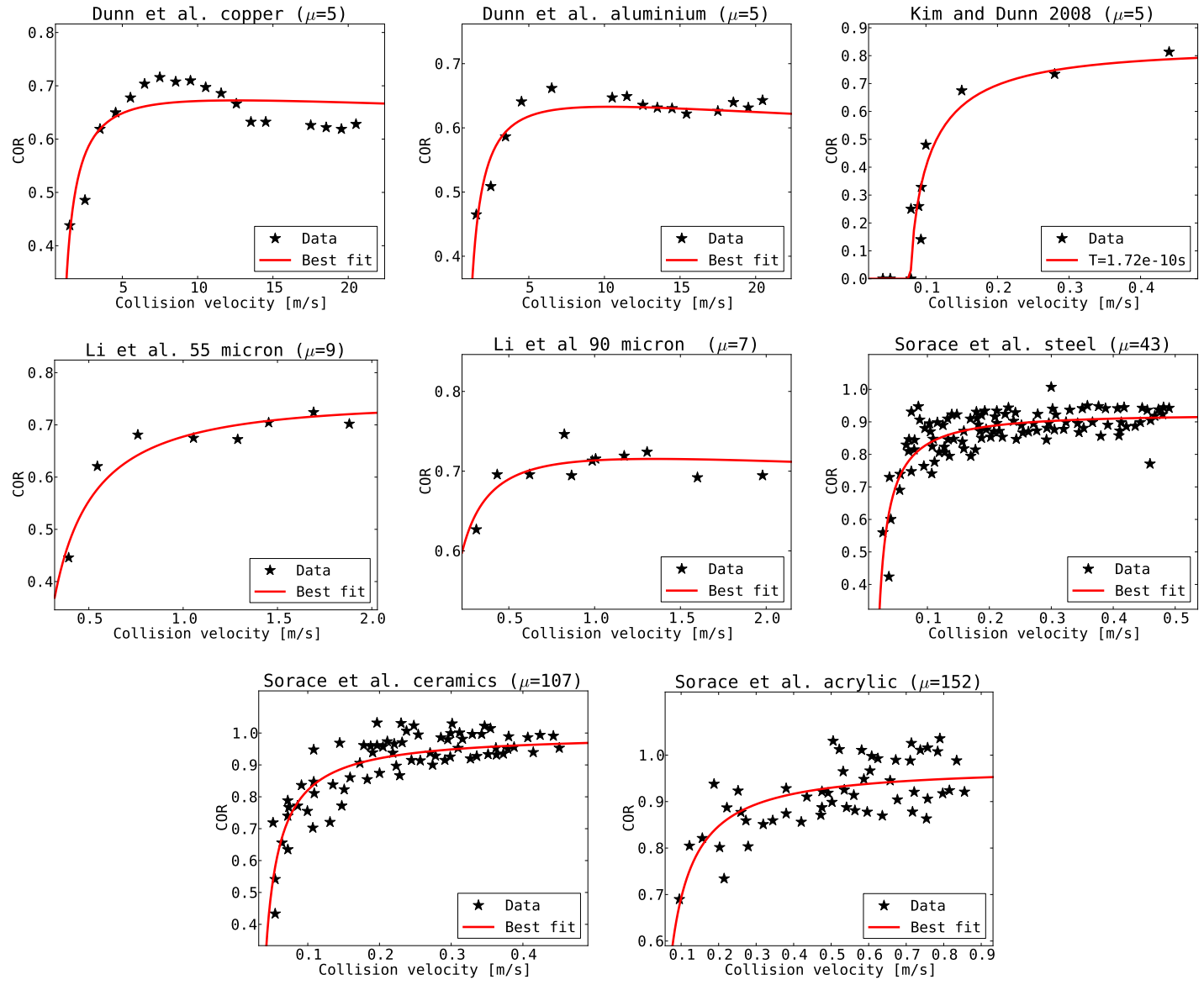

Figure C3: (continued) Best fitting models for the complete set of experiments. Note that the scale on the vertical axis varies. The value of the Tabor parameter $\mu$ (section 5) is shown above each plot. 
Table C1: Fit parameters obtained in section 4. The experiments are ordered from largest (top) to smallest reduced radius.

\begin{tabular}{|c|c|c|c|}
\hline Experiment & $\gamma\left(\mathrm{J} \mathrm{m}^{-2}\right)$ & $Y(\mathrm{MPa})$ & $T_{\text {vis }}(\mathrm{s})$ \\
\hline Sorace et al. acrylic & $0.48_{-0.43}^{+14.52}$ & $>67$ & $<8 \times 10^{-8}$ \\
\hline Sorace et al. ceramics & $37.8_{-30.8}^{+62.2}$ & $>2980$ & $<5 \times 10^{-9}$ \\
\hline Sorace et al. steel & $5.47_{-3.47}^{+18.53}$ & $>2040$ & $8.90_{-8.80}^{+13.10} \times 10^{-9}$ \\
\hline Li et al. 90 micron & $1.49_{-0.59}^{+0.71}$ & $>3130$ & $1.12_{-0.17}^{+0.28} \times 10^{-9}$ \\
\hline Li et al. 55 micron & $2.74_{-0.74}^{+0.76}$ & $>2670$ & $5.08_{-1.08}^{+1.92} \times 10^{-10}$ \\
\hline Kim and Dunn & $0.39_{-0.19}^{+0.61}$ & $>464$ & $1.72_{-1.62}^{+2.78} \times 10^{-10}$ \\
\hline Dunn et al. aluminium & $0.97_{-0.22}^{+0.28}$ & $>2490$ & $5.16_{-0.66}^{+0.84} \times 10^{-11}$ \\
\hline Dunn et al. copper & $1.34_{-0.59}^{+0.46}$ & $>3050$ & $3.90_{-0.90}^{+1.10} \times 10^{-11}$ \\
\hline Dunn et al. coated steel & $2.55_{-0.85}^{+0.95}$ & $>3320$ & $1.03_{-0.53}^{+0.67} \times 10^{-11}$ \\
\hline Dunn et al. steel & $2.42_{-0.92}^{+0.98}$ & $>3310$ & $1.55_{-0.85}^{+0.95} \times 10^{-11}$ \\
\hline Dunn et al. tedlar & $0.21_{-0.06}^{+0.06}$ & $>245$ & $1.70_{-0.20}^{+0.60} \times 10^{-10}$ \\
\hline Wall et al. tedlar & $0.16_{-0.04}^{+0.04}$ & $>90$ & $8.85_{-2.85}^{+5.15} \times 10^{-11}$ \\
\hline Wall et al. mica & $0.13_{-0.02}^{+0.02}$ & $192_{-12}^{+28}$ & $9.79_{-1.79}^{+1.21} \times 10^{-11}$ \\
\hline Wall et al. silicon & $0.17_{-0.03}^{+0.05}$ & $153_{-23}^{+22}$ & $5.97_{-2.47}^{+2.03} \times 10^{-11}$ \\
\hline Wall et al. molubdenum & $0.15_{-0.05}^{+0.07}$ & $>120$ & $5.74_{-3.74}^{+3.26} \times 10^{-11}$ \\
\hline Dahneke PVT & $0.11_{-0.10}^{+0.12}$ & $>280$ & $3.07_{-1.77}^{+1.93} \times 10^{-12}$ \\
\hline Dahneke PSL & $0.11_{-0.02}^{+0.03}$ & $>271$ & $2.14_{-0.64}^{+1.11} \times 10^{-12}$ \\
\hline
\end{tabular}

